\title{
Kadowaki-Woods ratio for strongly coupled Fermi liquids
}

\author{
Takuya Okabe* \\ Faculty of Engineering, Shizuoka University, 3-5-1 Johoku, Hamamatsu 432-8561, Japan
}

(Received 6 April 2007; revised manuscript received 1 October 2007; published 30 November 2007)

\begin{abstract}
On the basis of the Fermi liquid theory, the Kadowaki-Woods ratio $A / \gamma^{2}$ is evaluated by using a first principles band calculation for typical itinerant $d$ - and $f$-electron systems. It is found as observed that the ratio for the $d$-electron systems is significantly smaller than that for the normal $f$ systems, even without considering their relatively weak correlation. The difference in the ratio value comes from different characters of the Fermi surfaces. By comparing Pd and $\mathrm{USn}_{3}$ as typical cases, we discuss the importance of the Fermi-surface dependence of the quasiparticle transport relaxation.
\end{abstract}

DOI: 10.1103/PhysRevB.76.193109

PACS number(s): 71.10.Ay, 71.27.+a, 72.15.-v, 71.20.Be

It is widely known as a universal feature of heavy fermion systems that there holds the Kadowaki-Woods $(\mathrm{KW})$ relation $A / \gamma^{2} \simeq 1 \times 10^{-5} \mu \Omega \mathrm{cm}(\mathrm{mol} \mathrm{K} / \mathrm{mJ})^{2}$ between the electronic specific heat coefficient $\gamma$ of $C=\gamma T$ and the coefficient $A$ of the resistivity $\rho=A T^{2}$ in the clean and low temperature limit. ${ }^{1}$ According to the Fermi liquid theory, this is interpreted as an indication of the fact that $A$ is squarely proportional to quasiparticle mass enhancement due to strong electron correlation. On the other hand, transition metal systems have been reported before to obey a similar relation with a more than an order of magnitude smaller value of $A / \gamma^{2} .^{2,3}$ In view of the observation that there seems to exist several types of systems in this regard, the recent finding by Tsujii et al. ${ }^{4}$ is quite impressive that many $\mathrm{Yb}$-based compounds show the $\mathrm{KW}$ ratio $A / \gamma^{2}$ as small as the transition metals. Kontani derived the small ratio as a result of the large orbital degeneracy of the $4 f^{13}$ state of trivalent $\mathrm{Yb}$ by applying the dynamical mean field approximation to a periodic Anderson model of an orbitally degenerate $f$-electron states coupled with a single conduction band. ${ }^{5}$

To discuss the KW ratio $A / \gamma^{2}$ and the many-body mass enhancement effect, a simple model is usually adopted at the cost of neglecting material specific individual factors. In the present work, we are interested in such an effect as caused by a system-dependent factor, that is, the Fermi-surface dependence of quasiparticle current relaxation. The system should have a large enough Fermi surface relative to the Brillouin zone boundary in order for the quasiparticle current to dissipate effectively into an underlying lattice through mutual quasiparticle scatterings. In other words, the effectiveness of the transport relaxation may depend on the size and shape of the Fermi surface. To investigate this point definitely, we discuss the quasiparticle transport by taking into account the momentum dependence of quasiparticle scattering on the basis of realistic band structures. This has been hampered so far by a task required for not so simple Fermi surfaces of many band systems as could be simply modeled analytically. In terms of fairly realistic energy bands obtained from a first principles calculation, we evaluate those quantities which are not affected severely by the electron correlation effect. The theory in use is essentially within the phenomenological Fermi liquid theory described by renormalized quantities, and unlike a model calculation, no bare microscopic quantities appear explicitly. Schematic results using simple abstract models have been given before, in which a tight binding square lattice model and a two-band model are investigated. ${ }^{6-8}$

For the ratio $A / \gamma^{2}$, we make use of the expression

$$
\frac{A}{\gamma^{2}}=\frac{9 \alpha F}{8 \pi e^{2}}=21.3 \alpha F a\left[\mu \Omega(\mathrm{mol} \mathrm{K} / \mathrm{mJ})^{2}\right],
$$

which corresponds to Eq. (4.11) in Ref. 7 where we set $a=4 \AA$ for the lattice constant. In what follows, we substitute a calculated value for $a$. Below, we follow how to derive $\alpha F$, where $\alpha$ is a coupling constant and $F$ is a factor determined by the Fermi surface.

Following a microscopic analysis of the quasiparticle transport with vertex corrections properly taken into account, ${ }^{9}$ we may derive a phenomenological linearized Boltzmann equation. ${ }^{7}$ Generalizing the theory to take a many-band effect into account, in the low temperature $T \rightarrow 0$, we end up with the equation

$$
v_{p \mu}^{i}=(\pi T)^{2} \sum_{p^{\prime}, k} W_{p p^{\prime} k}^{i j} \rho_{p^{\prime}}^{j} \rho_{p-k}^{i} \rho_{p^{\prime}+k}^{j}\left(l_{p \mu}^{i}+l_{p^{\prime} \mu}^{j}-l_{p^{\prime}+k \mu}^{j}-l_{p-k \mu}^{i}\right),
$$

where $v_{p \mu}^{i}$ and $\rho_{p}^{i}=\delta\left(\mu-\varepsilon_{p}^{i}\right)$ are the velocity component and the local density of state of the renormalized (massenhanced) quasiparticle with the crystal momentum $p$ in the $i$ th band. The superscripts $i$ and $j$ are the band indices, while the subscript $\mu=x, y, z$ are Cartesian coordinates. In the right hand side of Eq. (2), the second to fourth terms in the parenthesis represent vertex corrections in the microscopic formulation. In terms of the solution $l_{p \mu}^{i}$, which physically represents stationary deviation of the Fermi surface in an applied electric field $E_{\mu}$, the conductivity is given by

$$
\sigma \equiv \sigma_{\mu}=2 e^{2} \sum_{p, i} \rho_{p}^{i} v_{p \mu}^{i} l_{p \mu}^{i}
$$

Equations (2) and (3) correspond to Eqs. (3.10) and (3.15) of Ref. 8, respectively. We may suppress the index $\mu(=x)$ in Eq. (3) as we discuss the cubic systems in what follows.

Instead of solving the simultaneous matrix equations [Eq. (2)] exactly, we use trial functions for $l_{p \mu}^{i}$ as commonly applied in a variational principle formulation of the transport problems. ${ }^{10}$ Assuming 


$$
l_{p \mu}^{i} \propto e_{p}^{i} \equiv \frac{v_{p \mu}^{i}}{\left|v_{p \mu}^{i}\right|},
$$

we obtain

$$
\alpha F \equiv \frac{\sum_{i, j} \alpha^{i j} c_{i, j}}{\left(\rho^{2}\left|v_{x}\right|\right)^{2}},
$$

where

$$
c_{i, j}=\sum_{\substack{k_{1}, k_{2}, k_{3}, k_{4} \\ k_{1}+k_{2}=k_{3}+k_{4}}} \rho_{k_{1}}^{i} \rho_{k_{2}}^{j} \rho_{k_{3}}^{j} \rho_{k_{4}}^{i}\left(e_{k_{1}}^{i}+e_{k_{2}}^{j}-e_{k_{3}}^{j}-e_{k_{4}}^{i}\right)^{2} / 4 \rho_{i} \rho_{j},
$$

and

$$
\rho\left|v_{x}\right| \equiv \sum_{i, p} \rho_{p}^{i}\left|v_{p x}^{i}\right| .
$$

We define coupling constants $\alpha^{i j}=\rho_{i} \rho_{j}\left\langle W^{i j}\right\rangle / \pi$, where $\rho_{i}=\Sigma_{p} \rho_{p}^{i}$ is the density of states of the $i$ th band at the Fermi level and $\left\langle W^{i j}\right\rangle$ denotes the quasiparticle scattering probability $W_{p p^{\prime} k}^{i j}$ averaged over the momenta $p, p^{\prime}$, and $k$. As the double sum in Eq. (2), dominated by the Umklapp processes, covers a complicated shaped phase space over the Fermi surface, it is generally a good approximation to take $W_{p p^{\prime} k}^{i j}$ out of the momentum sum as an averaged quantity. The total density of states $\rho=\sum_{i} \rho_{i}$ is substituted for $\gamma=2 \pi^{2} \rho / 3$.

In heavy fermion systems, the momentum dependence of $W_{p p^{\prime} k}^{i j}$ could be generally neglected; for the quasiparticle scattering, $W_{p p^{\prime} k}^{i j}$ is primarily caused by strong on-site Coulomb repulsion $U$. Then, we can make an order of magnitude estimate of $\alpha^{i i}$ in terms of Landau parameters $F_{0}^{i, s}$ and $F_{0}^{i, a}$. For an anisotropic Fermi liquid, as in an isotropic case, one can derive that the charge and spin susceptibilities are given by $\chi_{c}^{i}=2 \rho_{i} /\left(1+F_{0}^{i, s}\right)$ and $\chi_{s}^{i}=2 \rho_{i} /\left(1+F_{0}^{i, a}\right)$, respectively. Thus, for the systems in which charge fluctuations are suppressed, $\chi_{c}^{i} \rightarrow 0$, we obtain $F_{0}^{i, s} \gg 1$. On the other hand, in terms of $A_{0}^{i, s}=F_{0}^{i, s} /\left(1+F_{0}^{i, s}\right)$, one obtains a rough estimate of the coupling $\alpha^{i i}=\frac{1}{4}\left[\left(A_{0}^{i, s}-A_{0}^{i, a}\right)^{2}+\frac{1}{2}\left(A_{0}^{i, s}+A_{0}^{i, a}\right)^{2}\right]$. Therefore, under the normal condition that the spin enhancement is moderate, $\left(1+F_{0}^{i, a}\right)^{-1} \sim 1, \alpha^{i i}$ should universally stay around a constant of an order of unity. ${ }^{7}$ This corresponds to the condition to make the Wilson ratio $R_{W}=2$ in the impurity model. ${ }^{11,12}$ We discuss a normal state that the system is well away from critical instabilities, around which $A / \gamma^{2}$ will be strongly enhanced at variance with experimental results under consideration. ${ }^{13,18}$ We evaluate $F$ numerically for $\alpha=\alpha^{i j}=1$ to obtain $A / \gamma^{2}$ and investigate the Fermi-surface dependence.

It is noted that the factor $F$ is determined by the shape and extent of the Fermi surfaces relative to the Brillouin zone boundary. Microscopically, the mass enhancement due to the many-body effect is represented by the $\omega$ derivative of the electron self-energy $\Sigma(q, \omega)$ or by the renormalization factor $z_{p}^{i}$ as $\rho_{p}^{i}=\rho_{0, p}^{i} / z_{p}^{i}$, where $\rho_{0, p}^{i}$ is a bare density of states. It is easily checked that the factor $z$ cancels in $F$ when $z_{p}^{i}$ is independent of $i$. Otherwise, in case that a dominant contribu-
TABLE I. Calculated results.

\begin{tabular}{lccccc}
\hline \hline & $\begin{array}{c}a \\
(\AA)\end{array}$ & $\rho\left|v_{x}\right|^{\mathrm{a}}$ & $F$ & $N$ & $A / \gamma^{2 \mathrm{~b}}$ \\
\hline $\mathrm{USn}_{3}$ & 4.60 & 3.1 & 4.0 & 3 & 0.39 \\
$\mathrm{UIn}_{3}$ & 4.61 & 4.9 & 1.6 & 3 & 0.16 \\
$\mathrm{UGa}_{3}$ & 4.24 & 3.9 & 2.5 & 3 & 0.23 \\
$\mathrm{Pd}$ & 3.86 & 7.4 & 0.23 & 3 & 0.019 \\
$\mathrm{Pt}$ & 3.91 & 8.4 & 0.15 & 4 & 0.012 \\
\hline \hline
\end{tabular}

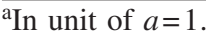

bIn unit of $10^{-5} \mu \Omega \mathrm{cm}(\mathrm{mol} \mathrm{K} / \mathrm{mJ})^{2}$.

tion to the resistivity comes from an electron-correlated main band, then the other bands may be neglected and $A / \gamma^{2}$ becomes independent of $z$ of the main band. As we see below numerically, it is found indeed that $F$ is dominated by a few scattering channels within a main band or two. Hence, we elaborate on a numerical estimate of $F$ on the basis of a realistic band calculation reproducing reliable Fermi surfaces of relevant bands, even if it may not take into account the local many-body correlation effects fully enough for the renormalized quantities such as $\rho_{i}$ and $v_{p}^{i}$ to be separately compared with experiments. As a matter of course, we must exclude the extreme case in which strong correlation modifies electron states around the Fermi level qualitatively from those of a band calculation. We apply our theory to those itinerant electron systems in which correlation strength is not negligible but not so strong.

To calculate $F$ for some typical cubic $d$ and $f$ itinerant electron systems in the fcc and $\mathrm{Cu}_{3} \mathrm{Au}$ structures, we have performed $a b$ initio band calculations within density functional theory using the plane wave pseudopotential code VASP with the Perdew-Wang 1991 generalized gradient approximation to the exchange correlation functional $E_{\mathrm{xc}} \cdot{ }^{14-17}$ By minimizing the total energy, we obtain the lattice constant $a$, which is accurate enough to be used in Eq. (1).

To evaluate $F$ numerically, we have to broaden the delta function $\rho_{p}^{i}=\delta\left(\mu-\varepsilon_{p}^{i}\right)$ by $\Delta$ to pick up electron states around the Fermi level. The width $\Delta$ of the order of real temperature should be decreased as the number of the $k$ points is increased until we confirm to have a convergent result. For the number $L$ of subdivisions along reciprocal lattice vectors, band calculations are performed with $L_{\text {band }} \sim 50$, from which we obtain the band energies $\varepsilon_{k}^{i}$ on the finer $k$ mesh of $L \sim 200$ by interpolation. As the fourfold $k$ sum in the numerator of Eq. (4), especially for the most important terms coming from the main $d$ or $f$ correlated bands, constitutes the most time consuming part of the calculation, we have to reduce the numerical task by some symmetry considerations not only on the cubic symmetry of the quasiparticle states but also on the relative directions of the four momentum vectors of the scattering quasiparticle states and the $x$ direction of the current flow. The reduction is particularly effective for the intraband scatterings $i=j$.

The calculated results are shown in Table I, where $F$ and $A / \gamma^{2}$ for $\alpha=\alpha^{i j}=1$ are shown along with the lattice constant $a$, the number $N$ of metallic bands contributing to the resistivity, and $\rho\left|v_{x}\right|$ defined in Eq. (6). We find that our results 


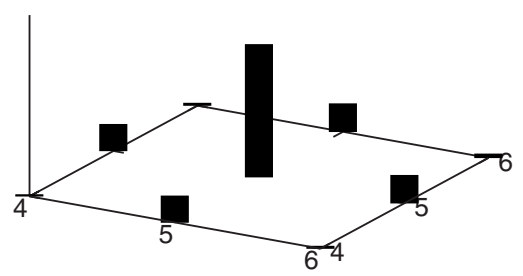

FIG. 1. $c_{i j}(i, j=4,5,6)$ for Pd. The contribution from the fifth band is dominant for the resistivity.

explain well the experimental tendency of an order of magnitude small values of the ratio $A / \gamma^{2}$ for the transition metal systems. As for the absolute values of the ratio, our results are a few times smaller than those observed evenly, but the accuracy of this order should not be taken seriously here. Among other things, the results indicate that different characters of the Fermi surfaces play an important role.

To show the relative contribution to the resistivity from relevant bands, relative magnitudes of $c_{i, j}$ in the numerator of Eq. (4) are shown for Pd and $\mathrm{USn}_{3}$ in Figs. 1 and 2, respectively. For Pd, the contribution to $F$ comes from the fourth to sixth bands, among which dominant is the fifth hole band of the $3 d$ character. Similarly, the fifth band contributes majorly not only to $\rho$, i.e., $\rho_{5} \simeq 5.4 \rho_{4} \simeq 12 \rho_{6}$, but also to $\rho\left|v_{x}\right|$ in Eq. (6). On the other hand, for $\mathrm{USn}_{3}$, while the 14th heavy electron band plays a central role, the 12th and 13th hole bands also make non-negligible contributions through the interband scatterings. Hence, as the first point to note, numerical importance of the interband contributions makes $F$ large in the $f$-electron system. This is partly because $\rho_{i}$ for $i=12,13,14$ are comparable with each other, namely, $\rho_{14} \simeq 2 \rho_{13} \simeq 3 \rho_{12}$. Moreover, it is remarked that the large and nearly spherical shapes of the Fermi surfaces are essential too. As the second point to note, the importance of the Fermi-surface geometry can be understood within a single band model by comparing contribution from the main band. We find that $c_{5,5} / \rho_{5}^{2}=0.097$ for Pd is an order of magnitude smaller than $c_{14,14} / \rho_{14}^{2}=0.93$ for $\mathrm{USn}_{3}$. The difference comes from the different characters of the Fermi surfaces.

According to an elementary formula $\sigma=e^{2} \rho v^{2} \tau=e^{2} \rho v l$, the conductivity $\sigma$ depends on $\rho v$ as well as $l$. In this context, the mean free path $l$ is not a single-particle property determined by a lifetime of the particle state, but it is the transport property which characterizes how efficiently the total electric current decays into a lattice system, e.g., in our case, through the mutual Umklapp scattering processes between the current carriers. In particular, regardless of interaction,

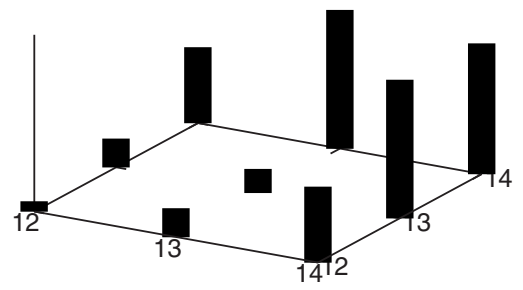

FIG. 2. $c_{i j}(i, j=12,13,14)$ for $\mathrm{USn}_{3}$. The interband contribution with the 14th band is important too.

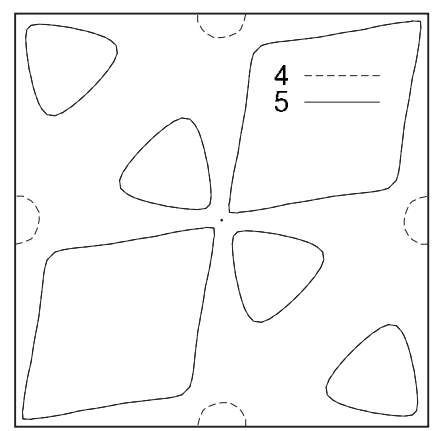

FIG. 3. The intersection of the Fermi surfaces of Pd $d$-hole states with the $(11 \overline{1})$ plane.

electrons in free space will not have resistivity. ${ }^{9}$ Thus, to evaluate the transport property $l$ correctly, it is crucial to take into account the momentum dependence of the scattering states and the conservation modulo of the reciprocal lattice vectors.

Note that $\rho\left|v_{x}\right|$ defined in Eq. (6) is related to the surface area $S$ of the Fermi surfaces, as $\rho d \varepsilon=S d k_{\perp} /(2 \pi)^{3}$. Hence, $\rho\left|v_{x}\right|$ too is independent of the mass renormalization $z$ as $F$ is, and for free electrons, we obtain $\rho\left|v_{x}\right| \propto k_{F}^{2} \propto n^{2 / 3}$. One can see a correlation between $F$ and $\rho\left|v_{x}\right|$ in Table I. In fact, Pd and Pt have twice as large $\rho\left|v_{x}\right|$ as the uranium compounds. The difference cannot be simply explained by the difference in the Fermi-surface volume $n$. It is caused by the fact that the $f$-electron systems have the nearly isotropic Fermi surfaces, while the $d$-electron systems have complicated ones with relatively large area compared to their total volume, as indicated in Figs. 3 and 4. The different characters of the surfaces affect not only the single-particle quantity $\rho\left|v_{x}\right|$ but also the transport property of the total current relaxation. As the order of magnitude difference in $F$ is not explained merely by $\rho\left|v_{x}\right|$, we have to resort to the other factor, that is, the transport property depending on the Fermi surfaces. It originates from the detailed $k$ dependence of the scattering states, as represented in $c_{i, j}$, or by the phase space volume available for all possible scattering channels under strict restrictions of energy and momentum conservations. Thus, our quantitative analysis concludes the important effect on the quasiparticle transport due to the shape and complexity of the Fermi surfaces.

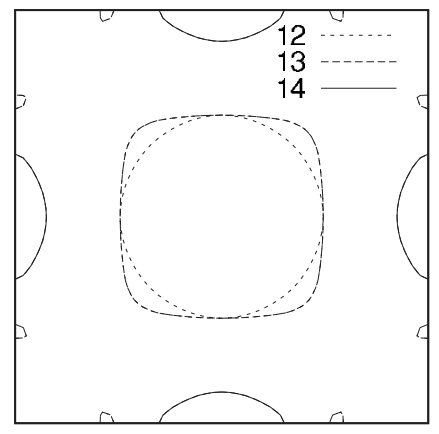

FIG. 4. The intersection of the Fermi surfaces of $\mathrm{USn}_{3}$ with the (100) plane. 
In summary, we evaluated the Kadowaki-Woods ratio $A / \gamma^{2}$ of some itinerant $d$ - and $f$-electron systems numerically on the basis of the Fermi liquid theory using quasiparticle Fermi surfaces obtained by band calculations. In a single framework, we find that the $d$-electron systems have smaller ratio than the $f$ systems, as observed, and among others, we pointed out an important effect to the transport coefficient $A$ originating from a commonly neglected specific feature depending on the characters of the Fermi surfaces. The effect is not understood fully as a single-particle property of interacting systems, but we stress the importance of the phase space

*ttokabe@ipc.shizuoka.ac.jp

${ }^{1}$ K. Kadowaki and S. B. Woods, Solid State Commun. 58, 507 (1986).

${ }^{2}$ M. J. Rice, Phys. Rev. Lett. 20, 1439 (1968).

${ }^{3}$ K. Miyake, T. Matsuura, and C. M. Varma, Solid State Commun. 71, 1149 (1989).

${ }^{4}$ N. Tsujii, H. Kontani, and K. Yoshimura, Phys. Rev. Lett. 94, 057201 (2005).

${ }^{5}$ H. Kontani, J. Phys. Soc. Jpn. 73, 515 (2004).

${ }^{6}$ T. Okabe, J. Phys. Soc. Jpn. 67, 2792 (1998).

${ }^{7}$ T. Okabe, J. Phys. Soc. Jpn. 67, 4178 (1998).

${ }^{8}$ T. Okabe, J. Phys. Soc. Jpn. 68, 2721 (1999).

${ }^{9}$ K. Yamada and K. Yosida, Prog. Theor. Phys. 76, 621 (1986). restriction due to momentum conservation in two-body scattering processes to dissipate a total electric current. In short, to realize effective dissipation, the system should have a large and regular shaped Fermi surface. In future, we will examine that the Fermi-surface dependent efficiency of mutual quasiparticle scatterings may depend on a type of transport current to be relaxed.

The author is grateful to N. Fujima, S. Kokado, and T. Hoshino for providing assistance in the numerical calculations. He also acknowledges computational resources offered from YITP computer system in Kyoto University.
${ }^{10}$ J. M. Ziman, Electrons and Phonons (Clarendon, Oxford, 1960).

${ }^{11}$ P. Nozières, J. Low Temp. Phys. 17, 31 (1974).

${ }^{12}$ K. Yosida and K. Yamada, Prog. Theor. Phys. 53, 1286 (1975).

${ }^{13}$ T. Takimoto and T. Moriya, Solid State Commun. 99, 457 (1996).

${ }^{14}$ G. Kresse and J. Furthmüller, Comput. Mater. Sci. 6, 15 (1996).

${ }^{15}$ G. Kresse and J. Furthmüller, Phys. Rev. B 54, 11169 (1996).

${ }^{16}$ G. Kresse and D. Joubert, Phys. Rev. B 59, 1758 (1999).

${ }^{17}$ J. P. Perdew, J. A. Chevary, S. H. Vosko, K. A. Jackson, M. R. Pederson, D. J. Singh, and C. Fiolhais, Phys. Rev. B 46, 6671 (1992).

${ }^{18}$ In three-dimensional nearly ferromagnetic metals, e.g., for $A_{q}^{\mathrm{RPA}}$ $=U /\left(\kappa^{2}+q^{2}\right)$ with $\kappa^{2}=1-U \chi_{0} \ll 1$, we obtain $\rho^{2}\left\langle\left(A_{q}^{\mathrm{RPA}}\right)^{2}\right\rangle_{q} \lesssim 1$ for $0.04 \lesssim \kappa \ll 1$. 